\title{
Enemy NPC Design Patterns in Shooter Games
}

\author{
Gabriel Rivera, Kenneth Hullett, Jim Whitehead \\ Augmented Design Lab \\ University of California, Santa Cruz \\ Santa Cruz, CA 9506 \\ gaariver@ucsc.edu, \{khullett, ejw\} @soe.ucsc.edu
}

\begin{abstract}
Gameplay in single-player shooter games primarily consists of combat with Non-player Characters (NPCs). However, there has been little research done to study how enemy NPCs affect player behavior in shooter games. As a consequence, there is no formal language for designers to discuss how NPCs are used in shooter games. This paper presents design patterns for NPCs in shooter games and explores their effects on gameplay. These patterns help designers communicate about and explore new ideas for enemy NPCs and further our understanding about how NPC types can be implemented, enabling more engaging experiences.
\end{abstract}

\section{Categories and Subject Descriptors K.8 [Personal Computing]: Games.}

\section{General Terms}

Design, Human Factors

\section{Keywords}

game design, design patterns.

\section{INTRODUCTION}

The analysis presented here is based on commercially-available games in the shooter genre. Shooter games allow players to traverse expansive, 3-dimensional spaces where they interact with the environment and other entities primarily through combat.

This research is focused on the enemy NPCs that populate a given level. Enemy NPCs are controlled by the computer and are the main source of conflict during gameplay. NPCs are one of the elements a designer can use to craft the pacing, challenge, and tension of a level. Designers can control not only where the NPC is placed but also the NPC's scripted behavior, how they are equipped, their level of health, their level of armor, and other variables.

Designers consider all of these factors when creating a level. There may be a terminology that many designers can use in order to talk about this; for-instance people can reference boss battles or other encounters. However, there is currently no catalogued language with a consistent terminology that can be used for describing these concepts. Design patterns help solve this problem

Permission to make digital or hard copies of all or part of this work for personal or classroom use is granted without fee provided that copies are not made or distributed for profit or commercial advantage and that copies bear this notice and the full citation on the first page. To copy otherwise, or republish, to post on servers or to redistribute to lists, requires prior specific permission and/or a fee.

DPG 2012, May 29, 2012, Raleigh, North Carolina, USA.

Copyright 2012 ACM 978-1-4503-1854-9/12/05... \$15.00. by creating a consistent terminology that can be referenced by people in the field. This results in people being able to communicate high level, overarching concepts faster to each other when design challenges come up. These concepts should be proven patterns that people know will work and that can be passed down to people newer to the field. By having defined patterns, it creates a lower barrier to entry for more complicated concepts to newer people in the field because there is a now a terminology that can be referenced and studied. Furthermore, patterns can help to explore new ideas when viewing a design challenge through all the different patterns. Overall, the introduction of design patterns to enemy NPC types would help to foster a more creative environment by allowing for clearer communication across designers of all levels.

For this research we explored elements that pertain to all NPCs within the shooter genre and then analyzed various games to see if NPCs consistently fell into patterns. Patterns were identified by observing NPC behavior and discerning which elements were combined in the same way within a number of games. Each pattern is accompanied by our observations about how it's used by designers to create gameplay, as well as a list of elements that define the pattern.

The primary contribution of this work is to create a common vocabulary for the different types of NPCs found in shooter games in order to label the different relationships that these NPCs have with the game play of the level. Through these patterns, a basic framework should be set for designers to collaborate and work off of in order to create better experiences for the player.

\section{RELATED WORKS}

There are two main fields of study that pertains to this research: design patterns and level design. The concept of creating a formalized language to describe a field was pioneered by Christopher Alexander in his book "A Pattern Language" [1]. The book revolved around architecture and how you could create a formalized language for how to approach architectural structures by classifying them as different patterns that were organized by their function, detailing how to solve each one. This was then expanded into the field of computer science in order to approach problems when organizing programs, as detailed in Design Patterns: Elements of Reusable Object - Oriented Software [3].

The concept of design patterns and how they relate to games has been explored by other sources. The first instance of this was Björk and Holopainen's Patterns in Game Design, which was the first foray into investigating how patterns were applied to the medium. As games research has expanded other people have begun looking at more specific patterns in game design. Hullett and Whitehead's Design Patterns in FPS Levels [5] investigates patterns in level design and how it affects a player's experience of the game. The patterns represented in this paper do affect NPCs 
and are mentioned briefly in section 6. However, due to time constraints it wasn't feasible to extensively examine how these Level Design Patterns (LDPs) affected the NPC patterns presented in this paper.

Other people have examined how NPCs affect a player's experience during combat. Mike Birkhead investigates NPCs within combat in Tips from a combat designer: The art of boss design, which examines the idea of boss encounters and how to design a NPC to fit one. Benson Russell's A Deeper Look Into The Combat Design of Uncharted 2 also investigates designing combat in the shooter genre with a small emphasis on NPC interaction. However these articles do not apply patterns to NPC design or combat within shooters. They examine the overall design and dissect what happens in games but don't try to develop a large language that can be used to communicate concepts to other designers with.

\section{ELEMENTS OF A NPC}

Below is a list of elements that make up a NPC as well as a brief description of how they can be used by a designer to create gameplay during combat. These will be used in the pattern collection to categorize the specific patterns.

Movement Type - This describes the way the NPC will typically move in a combat situation. Many NPCs employ multiple Movement Types and can switch between them depending on the situation.

- Flanking Intensive - The NPC will move to attack from unexpected directions, i.e. the NPC tries to approach the player from a different side than where the player's attention is directed.

- Passive - The NPC will not move when attacking. Never straying too far from that location and available cover.

- Slow Push - The NPC will slowly advance on the position of the opposing force, usually in a straight line. This can be without the need for cover, but it is possible for the NPC to utilize cover while making its way forward. This main difference between this and Cautious is that it will constantly try to close the distance from its target and not try to stay away.

- Rush - The NPC will make a dash at a specific target without any regard for their safety, typically in a straight line. However, the main aspect of this movement type is that they will attack very fast and often try to close the distance between themselves and their target as fast as possible.

- Cautious - When used, it means that the NPC is opting to move around the battlefield but tries to maintain a distance from its target. Often trying to utilize cover when possible and not closing the distance when possible. This is different from a slow push because this NPC tries to maintain a specific radius around its target, without advancing.

Movement Range - This is how far the NPC will move during an engagement. This can be Low, Medium, or High.

Movement Frequency - This is how often the NPC will change their position during an engagement. This can be Low, Medium, or High.

Attack Frequency - This describes how often the NPC will initiate an attack. This can be Low, Medium, or High.

Weapon Type - This general category utilizes the weapon patterns from Weapon Design Patterns in First-Person Shooter
Games [4] and can be referenced for further information on them. The patterns include the following:

- Sniping Weapon

- Close Blast

- Assault Weapon

- Projectile

- Power Weapon

- $\quad$ Melee Weapon

Weapon Damage - A general indicator on how much damage the NPC will do to the player's Health, Shields, or Armor. This can be Low, Medium, or High.

Armor/Health - This denotes how much damage the NPC can take before being killed. This will typically be linked to how hard the NPC is to defeat. This can be Low, Medium, or High.

Motive - This is an indicator of what type of combat encounter the NPC would create and shows its purpose to the designer. This hinges on three main factors that an NPC can affect:

- Challenge - The degree of difficulty within a combat encounter.

- Tension - The degree of mental stress the player experiences during a combat encounter.

- $\quad$ Pacing - The degree of movement that the player will engage in during a combat encounter.

A pattern can affect each of these three factors by creating a situation where they can be at Low, Medium, or High.

\section{STRUCTURE OF NPC DESIGN PATTERNS:}

- Name - The name of the pattern should describe what the pattern does essentially.

- Description - A brief description of how the pattern is typically used and the effect it has on gameplay. This can be seen as the primary role of the enemy NPC.

- Affordances - This section lists the elements of the NPC and what those elements can be broken down into.

- NPC Relationships - Description of any special relationships with other patterns. This includes an Enemy NPC switching to a different pattern.

- $\quad$ Examples - Some examples from well-known games.

\section{PATTERN COLLECTION}

Below is a list of all the patterns that we have collected during our research. Each base pattern specifies the primary function of that general type, while each sub pattern denotes how that function is carried out.

- $\quad$ Soldier - Grunt, Elite, Grenadier, Sniper

- $\quad$ Aggressor - Suicide, Swarm, Berserker

- Carrier - Sacrifice, Summoner

- $\quad$ Tank - Turret, Shield

The following sections detail all of the base patterns and at least one of their sub patterns. Not all patterns mentioned above are presented here due to the constraints on the length of the paper. For a more detailed description of these patterns and all associated sub patterns, please visit the Level Design Patterns website at ldp.soe.ucsc.edu. 


\subsection{Soldier}

Soldier is a NPC that will pressure the player from long range. Its main strategy is to control the available space in the encounter. NPCs of this type make up the majority of units during an encounter. They are primarily used to control pacing by forcing the player to take particular paths through the environment. These NPCs will have a weapon type that is an Assault, Close Blast, Sniping, or Projectile.

\subsubsection{Grunt}

\section{Description:}

The Grunt is a weak NPC that will try to maintain a medium distance away when attacking. The main function this serves is to draw the player to forward through the level and increase the player's confidence. This pattern is distinguished by always having medium movement range, medium movement frequency, and light armor. The motive of the Grunt pattern is to create a situation with low tension and low challenge.

\section{Affordances:}

- Movement type can be Slow Push, Flanking Intensive, or Cautious.

- Attack frequency can be either Low or Medium.

- Weapon damage can be either Low or Medium.

\section{NPC Relationships:}

The grunt has a special relationship with the Suicide pattern, because sometimes a grunt may change to the suicide pattern in the middle of an encounter.

\section{Examples:}

Halo: Combat Evolved - The Grunt is a small unit that appears in every game within the Halo franchise. It has a low amount of Armor and is usually to be equipped with an assault weapon that does a low (Plasma Pistol) or medium (Needler) amount of damage. They exhibit the special relationship with the Suicide pattern in that they will self-destruct in times of desperation. The range it keeps is either short or medium but tries to pester the player by implementing the Cautious movement type.

During the campaign they primarily occur within encounters to create a lower challenge but increase the pace of the encounter. As a consequence, the player feels more empowered and will pursue a route that contains a higher ratio of grunts compared to any other path. This occurs in the level The Pillar of Autumn; often the designers put grunts down a particular corridor to encourage the player to move in that direction. This signals to the player that it is the correct route to follow while lowering challenge, increasing the pace, and lowering player tension.

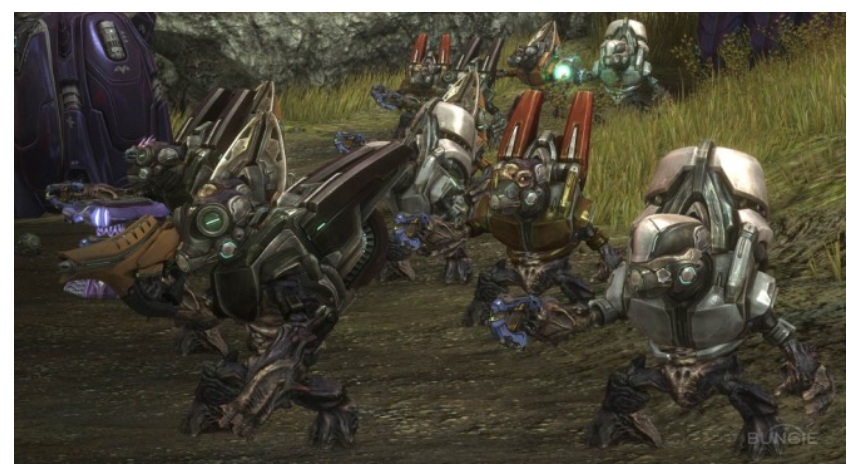

Figure 1: A Group of Grunts in Halo: Reach
Half-Life 2 - The Metro Police Officer utilizes a Slow Push or Cautious Movement Type and primarily is equipped with an assault weapon, typically a sidearm. They will shift between the movement types in an effort to move a player forward. Typically this means that they will begin in a cautious movement type and, if they player doesn't pursue them, will move toward the player in order to get the player to move. This doesn't occur in any particular instance but can be seen where there are Metro Police Officers in levels such as Route Kanal or Water Hazard. In the game, they basically act as bait to simply pull the player forward. They are primarily seen as the main enemy in the early game and are increasingly used as bait in the latter half of the game.

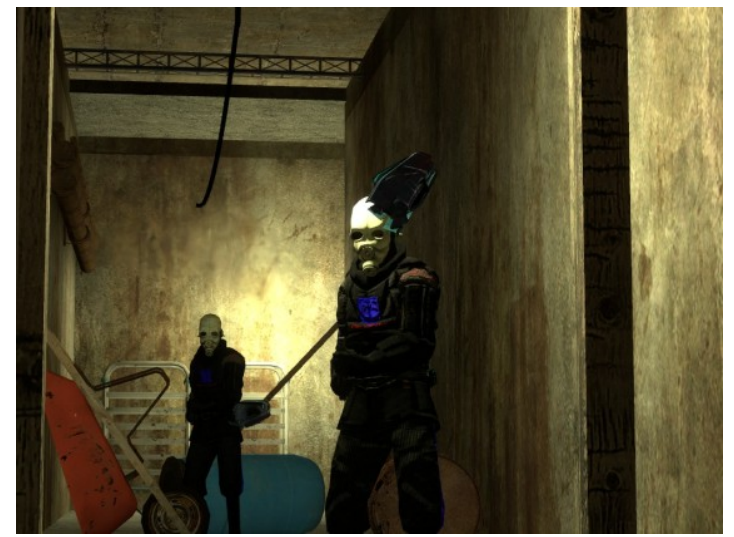

Figure 2: Two Metro police officers in Half-Life 2

\subsubsection{Grenadier \\ Description:}

The Grenadier NPC pattern distinguishes itself by being a weaker enemy that tries to stay a decent distance away in order to fire projectile weapons at the player. The main function that this NPC pattern is designed to do is to encourage the player to move through pressure made by the explosives. This serves as the antithesis of the Grunt NPC Pattern because this pattern increases challenge and tension to encourage the player to move as opposed to lowering it. The main traits that are required to distinguish this class are that they have a medium movement range, low movement frequency, a medium attack frequency, and a projectile weapon type. The Motive of this pattern is to create a situation with high tension and high challenge.

\section{Affordances:}

- The Range can be either medium or long.

- The Movement Type can be Passive, Slow Push, or Cautious.

- The Armor can be either medium or low.

\section{NPC Relationships:}

None.

\section{Examples:}

Gears of War - The Boomer is a large unit that has a long range explosive weapon and medium grade body armor. It utilizes a slow push movement type and appears as a larger version of the typical locust drone soldier. It fires large explosives that force the player to evacuate their current cover position, unless they want to 
be hurt due to the splash damage. This increases the pace at which the player moves through the level by pressuring them.

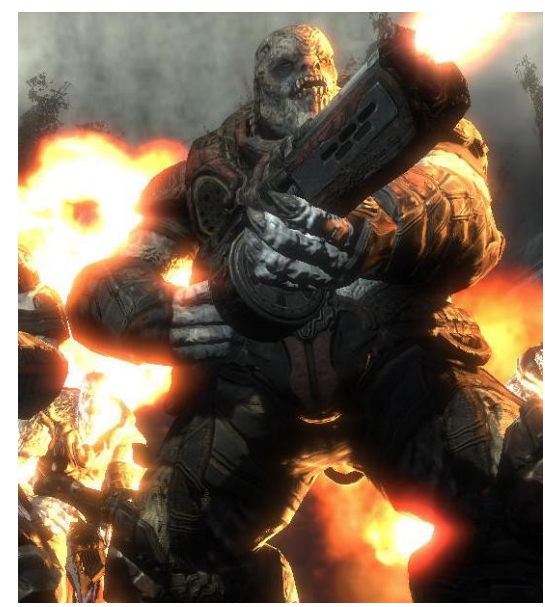

Figure 3: A Boomer in Gears of War

Uncharted 2: Among Thieves - A rocket soldier is a unit with a low amount of armor that maintains a passive movement type and deals with a projectile weapon type. It typically appears as a soldier in camouflage and bandanna that has an RPG with a band of ammo clips on its shoulder. It represents the atypical Grenadier, staying as far away as possible by forcing the player to move with high damage explosives. Thereby increasing the player's pace through the level without directly exposing itself to danger.

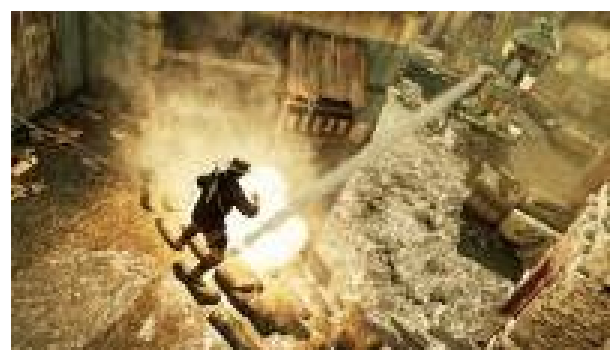

Figure 4: An allied NPC, Tenzin, is being shot by a rocket soldier from Uncharted 2: Among Thieves.

\subsection{Aggressor}

Aggressor is a NPC that will immediately close the distance to the player in order to attack them with a Melee or Close Blast weapon. Its main function is to increase challenge in an encounter by pressuring the player. This type of NPC complicates the player's situation by forcing them to deal with an immediate threat at close range. Its presence also serves to increase player tension, because there is an increased amount of pressure from the player to kill it before it starts to move in and attack. Every sub pattern of this type has the Rush movement type, high movement range, high movement frequency, high attack frequency, and a Melee or Close Blast weapon type.

\subsubsection{Berserker}

\section{Description:}

The Berserker is a sub pattern of the Rush NPC pattern and distinguishes itself by being an enemy that will rush the player to deal a high amount of damage over a prolonged amount of time. The main function of this NPC type is to give the player a high amount of challenge over a short period of time. The high amount of damage they do, forces the player to deal with them immediately. All patterns of this type will have a high amount of weapon damage and since it is a derivative of the Rush NPC pattern it keeps the same requirements to stay within the Rush NPC Pattern. All NPCs within this pattern can have armor of any level. The motive of the berserker pattern is to create a situation with High Challenge.

\section{Affordances:}

- There are no affordances in this NPC Type.

\section{NPC Relationships:}

None.

\section{Examples:}

Borderlands - The Psycho wears a mask and the lower portion of an orange jumpsuit. He rushes the player at a high speed and wields a large hatchet that deals a high amount of damage when it hits. The unit will frequently attack the player and will run across the entire battlefield in order to engage them. A couple of this unit type is usually put into larger groups of enemies in order to put more pressure the player more by having a more aggressive enemy.

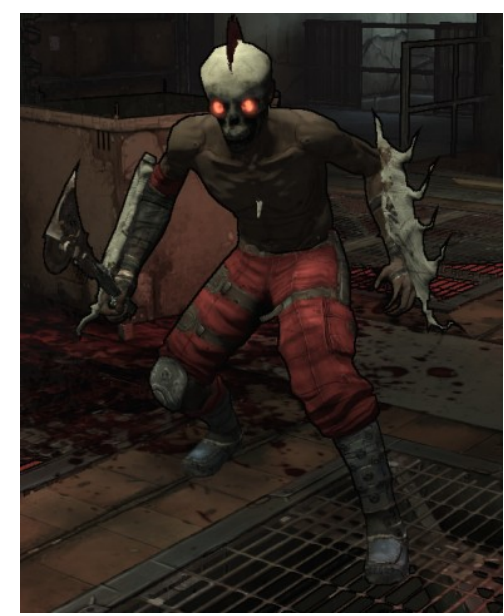

Figure 3: A Burning Psycho in Borderlands

Gears of War - The Butcher is a large unit that appears throughout the Gears of War franchise, it carries a cleaver that it uses to strike the player with and has medium armor. It has a low speed but it will try to engage the player and rush toward him from any area on the battlefield. He will repeatedly try to attack the player as long as he/she is within its sight. The large, imposing presence serves to make it immediately noticeable on the battlefield and it will consistently make players prioritize it first because of the high level of damage it does. 


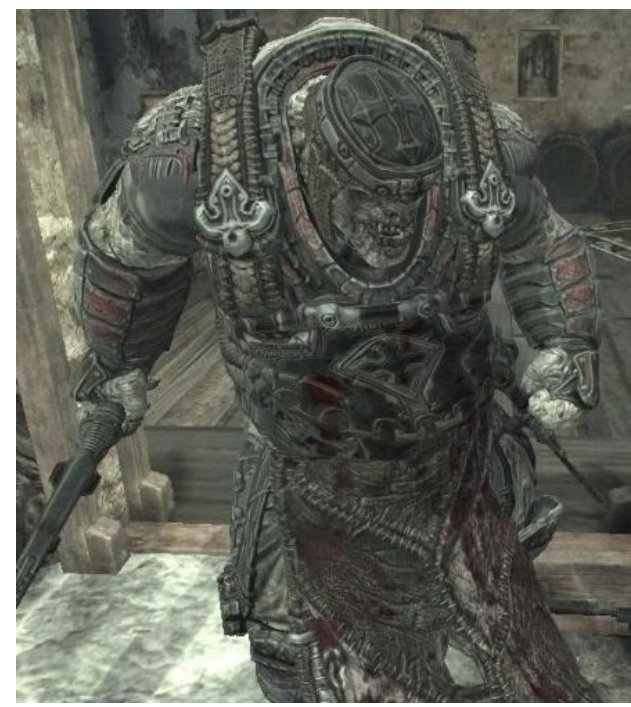

Figure 4: A Butcher from Gears of War

\subsection{Carrier}

Carrier is a NPC that will spawn more NPCs during an encounter. Their function is to increase the amount of tension that the player has by creating more enemies for the player to engage. The player knows that the number of enemies will keep increasing if the Carrier isn't dealt with. Therefore, the NPC's presence will be at the forefront of the player's mind throughout the battle since it is a threat that should be taken out early. Furthermore, the longer it stays alive, the more enemies the player with have to deal with; which increases the level of challenge. NPCs in this category will all have a high movement range, high movement frequency, and low armor. This is because the NPC doesn't want to get killed so there is a high movement frequency and range but designers want these to be killed fast so it's given a low amount of armor.

\subsubsection{Sacrifice}

\section{Description:}

Sacrifice is a sub pattern of the Carrier NPC pattern and is mainly distinguished by its ability to spawn enemies around its body upon death. The main function of this NPC is to increase the amount of tension within an encounter because the player will know that as soon as this NPC dies then they will immediately have to deal with more enemies. The common elements within all units of this pattern are a rush movement type, a high attack frequency, and a projectile weapon type. The motive of this pattern is to create a situation with a high amount of tension.

\section{Affordances:}

- None

\section{NPC Relationships:}

This NPC pattern can spawn NPCs of the suicide and swarm patterns.

\section{Examples:}

Dead Space - The Pregnant Necromorph appears in Dead Space 1 and 2. It will explode when shot and maintains a low amount of armor. It spawns smaller swarming units when it is hit; furthermore, it rushes the player in hopes that it will explode close by. It will appear solo in the game but occasionally appears in larger heterogeneous groups. The threat of creating a larger amount of smaller enemies increases the player's tension level, since the player will only release more enemies when they kill the pregnant necromorph.

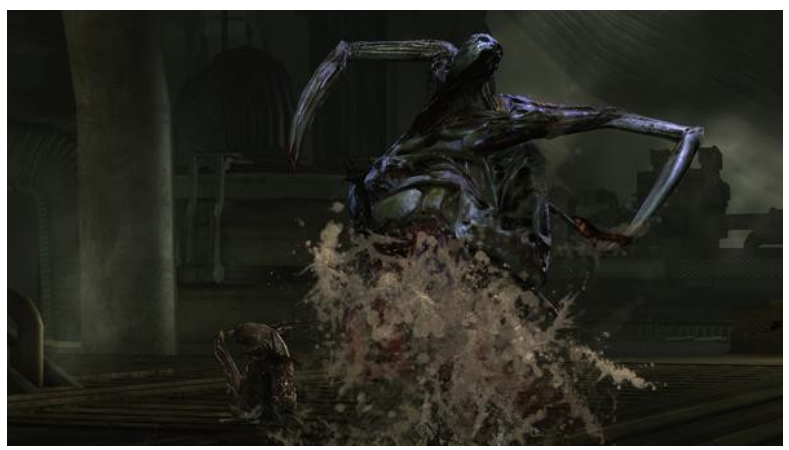

Figure 5: A pregnant necromorph spawning units after being killed in Dead Space.

Halo: Combat Evolved - The Carrier Flood form is a carrier that has a low amount of armor and spawns units through an explosive weapon type at close range. It typically tries to assault the player in the middle of a battle when in a larger heterogeneous group. It increases the tension of theleve because the player knows it can inflict a high amount of damage if it explodes nearby and only serves to create more enemies. This creates a situation where the player has to choose the best time to eliminate it in order to mitigate damage.

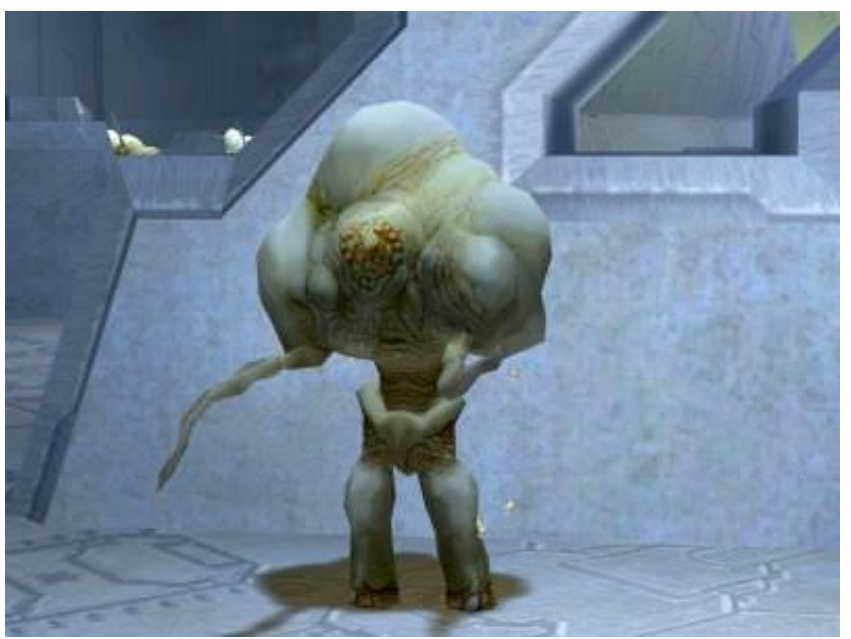

Figure 6: A Flood Carrier in Halo: Combat Evolved

\subsection{Tank}

Tank is a NPC that will raise the Tension and Challenge for the player. This occurs because the tank poses a significant singular threat that is required to be beaten in order to progress. Tank NPCs are meant to slow player progress by being hard to overcome, which will slow the pacing of the game. As a consequence, this pattern's main distinguishing feature is the NPC's ability to take a large amount of damage through an enormous amount of armor or health.

\subsection{Turret \\ Description:}


The Turret NPC Pattern is a sub pattern of the Tank NPC pattern and is mainly distinguished in how it is a slow moving NPC that will cause an extremely high amount of damage at long range. The main function of this NPC is to increase the amount of challenge and tension for the player by creating a relatively stationary threat to the player that is hard to remove. The common elements in this pattern are that all NPCs have a passive movement type, a high movement range, low movement frequency, high attack frequency, long range, and a power weapon as its weapon type. The motive behind this pattern is to create a situation with a high amount of tension, high amount of challenge, and a medium pacing.

\section{Affordances:}

- None

\section{NPC Relationships:}

This NPC pattern will sometimes switch to the Berserker NPC pattern for a period of time.

\section{Examples:}

Halo: Combat Evolved - The Hunter is a Turret unit that employs almost every single category for the unit type. This is because it utilizes attacks at long range with its beam cannon and then falls to short range to melee attacks with its shield arm. It has an aggressive behavior as it will continuously assault the player, has a high armor value to make it hard to kill, and all of its attacks do a large amount of damage. Finally, this unit travels in pairs. The reason for this is primarily because they have weak points on their backs so they will cover each other. The Hunter provides a large challenge due to its powerful weapon and high armor. The tension comes when a player is forced to try and kill one during a level, it is only increased by the fact that they appear in pairs.

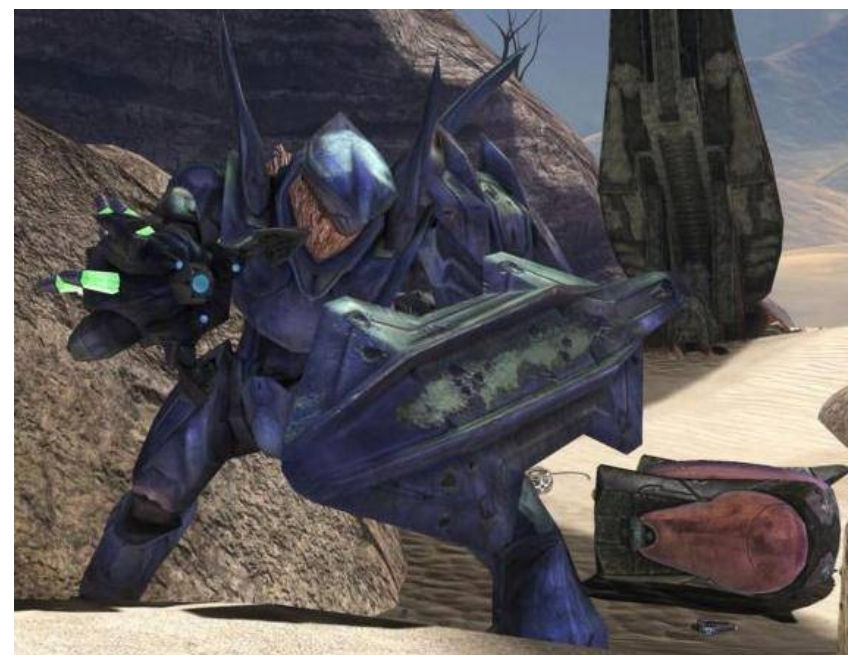

Figure 7: A Hunter from the Halo franchise

Call of Duty: Modern Warfare 2 - The Juggernaut is a tank that employs a power weapon at long range. It maintains a passive movement type that slowly follows the player from any part of the battlefield but remains stationary when the player is in sight. It also has a very high amount of armor. It appears as a very large, heavily armored man with a white mask who is armed with a heavy machine gun. The Juggernaut provides a large challenge that the player has to overcome and so a large amount of tension will automatically develop. The fact that it's so hard to remove only increases this effect.

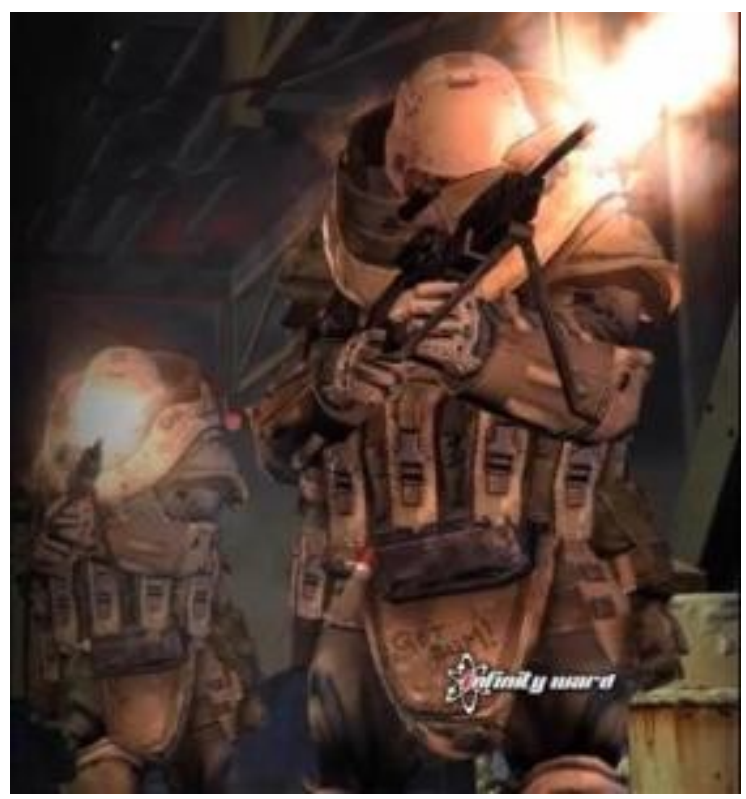

Figure 8: Two Juggernauts from Call of Duty: Modern Warfare 2

\subsubsection{Shield}

Description:

The Shield NPC is a sub pattern of the Tank NPC pattern and is mainly distinguished in how it has a high amount of armor but only in one particular direction. Their main function is to increase challenge and tension by creating a large, almost unstoppable force that slowly closes in on the player. The increase in challenge is because the player is then forced to rethink their strategy and flank in order to kill it. The increase in tension is derived from the fact that this NPC type will close in very slowly but is steadily getting closer to killing the player. The common elements shared in the NPC pattern are that it has a slow push movement type, a high movement range, and a medium movement frequency. The motive of a Shield NPC is to create a situation with a medium amount of challenge, high amount of tension, and high amount of pacing.

\section{Affordances:}

- The weapon type can be a side arm or assault.

\section{NPC Relationships:}

None.

\section{Examples:}

Halo: Combat Evolved - The Jackal is an example of a shield NPC because it has a high amount of armor where its shield is located and no damage is taken. However, the rest of the unit's body has a very low amount of armor resulting in numerous weak spots that its shield doesn't protect. The weapon that it uses is fairly short range so it must close the distance in order to do more damage. It does a relatively low amount of damage to the player as well and travels in either homogenous teams or heterogeneous teams; however it will never venture out alone. It is primarily used as a means to give more pressure to the player from a particular direction. 


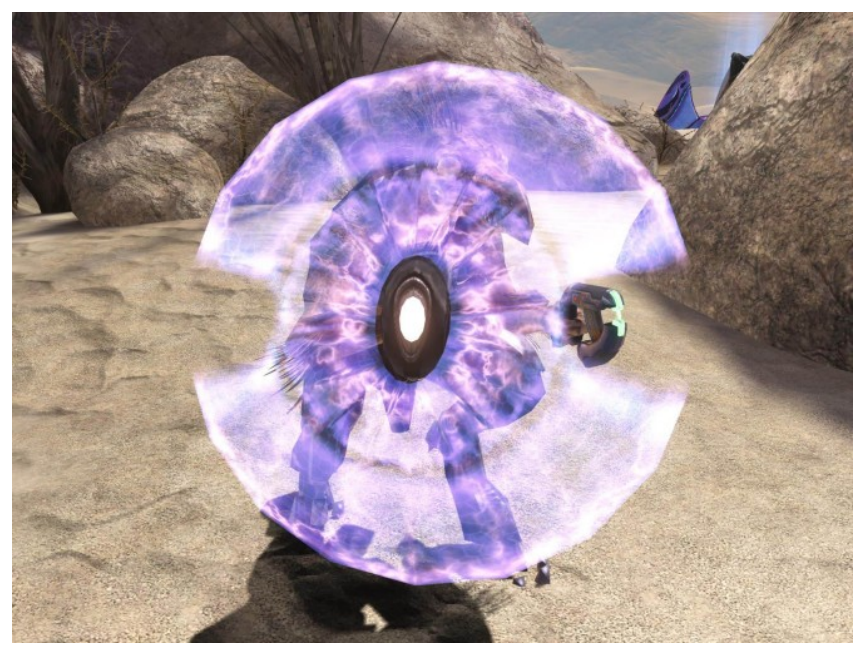

Figure 9: A Jackal in the Halo Franchise

Uncharted 2: Among Thieves - The turtle NPC appears as a military personnel that is equipped with a riot shield that blocks all damage from the front. Its primary method of offense is a machine pistol that can only be used at close range. It will engage the player from anywhere across the battlefield and will slowly try to close distance to the player's position (the more fire it's under the slower it goes). It has a medium movement frequency because it will pause from time to time. This unit serves to increase the tension in the player because it will move faster toward the player the less it's shot at. However, a player can only kill it by maneuvering around it and therefore not keeping it preoccupied. This situation creates a high amount of challenge that forces the player to continuously keep an eye on the unit.

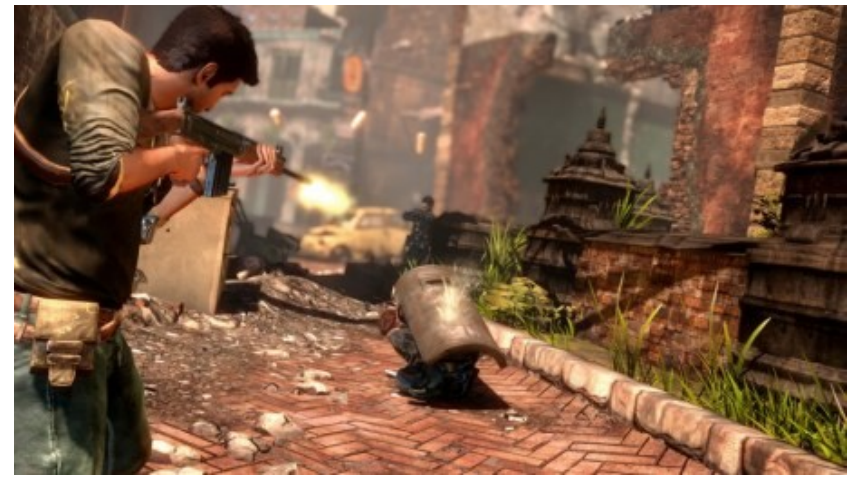

Figure 10: A turtle unit getting attacked by the player in Uncharted 2: Among Thieves

\section{EXAMPLE ANALYSIS}

To show the usefulness of NPC design patterns we will use them to analyze a short encounter and generate a new enemy type. The level Winter Contingency in the game Halo: Reach contains an encounter in which the group is tasked with bringing a communications outpost back online. This sequence starts with the team landing in front of the communications outpost in order to secure the location.

After starting the level, the player encounters their first group of enemy NPCs in an Arena with Flanking Routes to the left and right. The NPCs that populate the arena are a small force of
Grunts and Jackals. This encounter has a low amount of challenge and allows the player to gain a foothold without much effort. It is fairly easy for the player to move forward and incapacitate the Grunts, which fall under the Grunt NPC pattern. However, it is much harder kill the Jackals in a head on attack since they are a part of the Shield NPC pattern. The interplay between the Grunt and Shield patterns help to create a much easier encounter for the player by driving them to explore the area and flank the Jackals.

The player goes into the encounter and immediately recognizes that most of the Jackals were located in the Arena, where the player is at a disadvantage. Since that place is the hardest to break through, the player is drawn to the left because the Grunts offer a lower level of resistance. The Grunts signal to the player this path is safer and encourages them to move through the Flanking Route. The player can now flank the exposed back of the Jackals, which has a pattern specific weakness of only being able to withstand a large amount of damage from one direction.

We can analyze this encounter and explain it through the enemy NPC patterns that we have created. The designers used Shield NPCs in order to bar the player's way from one direction and give the illusion of a higher degree of challenge. However, by adding in the Grunt NPCs it allowed them to encourage the player to move into an advantageous position. The interplay between these two types helped to create an encounter with a low amount of challenge but high amount of tension.

Creating a new NPC that could encompass the experience in the example above would require a NPC pattern that creates a high amount of tension but low challenge. Since there isn't a predefined pattern for this situation it forces us to come up with a new one. An NPC Pattern requires the Movement Type, Movement Range, Movement Frequency, Attack Frequency, Weapon Type, Weapon Damage, Armor/Health and Motive. The Motive is given because the primary reason to create the pattern is so there is an NPC that will create a situation that has a High amount of Tension but Low amount of Challenge. The Movement Type of the NPC can be the Cautious movement type because we want the enemy to last in the encounter, that way the tension remains. The Movement Range should be Medium to keep the enemy within the typical range of the player but it should remain far enough to stay alive. This keeps the possibility of tension but should lower the challenge by making it easier to hit.

The Attack Frequency should be low, that way the player doesn't always know where they are since they don't typically engage them. The aim here is to make it so that the player has the possibility to lose track of them, to increase the tension, and the infrequent attacks should decrease challenge by allowing the player to regenerate health or grab cover. The Weapon Type could be a Power Weapon with a High amount of weapon damage. This means that the player would want to be on the lookout for the enemy constantly due to the inherent danger of being shot but the prospect of obtaining the Power Weapon could cause them to actively seek it out. This high amount of damage might make the enemy too hard, so to balance this a low amount of armor and health is required. By making the enemy easy to kill it lowers the level of challenge again but the tension from its weapon isn't lost.

Finally, we have to categorize this new enemy. This enemy increases the tension and lowers the amount of challenge in an encounter. Since it has more to do with tension than challenge, it fits into the category of Carrier but it doesn't fit into the affordances that pattern allows. In this case, it would be more suitable to create new base pattern for this NPC pattern. 
This example should highlight how a new NPC can be created by using the framework laid out in the paper. By pursuing a specific emotional response from the player it created the opportunity to explore what the framework had to offer. This experiment exposed that there was a possible enemy NPC that didn't fit into the base patterns that were currently available. Thereby expanding the framework for new ideas and allowing new possibilities to emerge.

\section{CONCLUSION}

Patterns are tools for designers that enhance their capability to reason through a design challenge. In this case, the tools presented in this paper allow them to examine how they use NPCs in a level. It creates a situation where designers can now make decisions on how to implement NPCs in a level through consulting a defined methodology rather than relying solely on past experience. Furthermore, the patterns enable the designers to communicate their ideas and design decisions across the entire team in a clearer fashion. This creates a smoother process when designing a game and a more creative environment.

We hope that enemy NPC patterns will inspire designers to explore the uses of enemy NPCs and create new gameplay experiences. The patterns could be viewed as a toolkit that the designers can use as building blocks for each experience. This would empower them to explore different levels of user interaction by mixing NPC patterns within the same area to create combinations or teams for a desired effect. There's also the possibility of creating a permutation of an existing pattern to create a new NPC. Exploring these new NPC types would open up new possibilities for designers by expanding the toolbox at their disposal.

Future work in this area could include a more in-depth examination on how NPCs can affect players within the FPS level design patterns. This research can also be furthered through examining the relationships between the enemy NPC patterns presented in this paper.

Consequently, it would also be useful to expand the patterns to include friendly or neutral NPCs. By doing this, it might open up a more varied toolset for how to illicit a particular player reaction. For-instance, we could create more player tension by using more Shield NPC types or turning the battle into a hostage situation. If research into this area were to continue it would create a larger toolset for designers to pull from. Each of these tools would allow designers to have greater control on how to affect a player's emotional experience within a combat encounter.

\section{Games Cited}

Borderlands, Gearbox Software, 2009.

Call of Duty: Modern Warfare 2, Infinity Ward Inc., 2009.

Dead Space, EA Redwood Shores, 2008.

Gears of War, Epic Games Inc., 2006.

Half-Life 2, Valve Software, 2004.

Halo: Combat Evolved, Bungie Studios, 2001.

Halo 3: ODST, Bungie Studios, 2009.

Halo Reach, Bungie Studios, 2011.

Uncharted 2: Among Thieves, Naughty Dog, 2009.

\section{REFERENCES}

[1] Alexander, C. “A Pattern Language.” Oxford University Press. 1977.

[2] Björk, S., Holopainen, J. "Patterns in Game Design." Charles River Media. 2004

[3] Gamma, Erich, Richard Helm, Ralph Johnson, and John Vlissides. Design Patterns: Elements of Reusable Object Oriented Software. [S.1.]: Addison - Wesley, [...]. Print.

[4] Giusti, R., Hullett, K., and Whitehead, J.. "Weapon Design Patterns in First-Person Shooter Game." Under Review.

[5] Hullett and Whitehead. "Design Patterns in FPS Levels." In Proceedings of Foundations of Digital Games (FDG 2010). Monterey, California, USA. 19-21 June 2010.

[6] Birkhead, Mike. "Gamasutra - News - Tips from a Combat Designer: The Art of Boss Design." Gamasutra. 19 Jan. 2012.Web.27Feb.2012.

$<$ http://www.gamasutra.com/view/news/129150/Tips_from_a _combat_designer_The_art_of_boss_design.php $>$.

[7] Russell, Benson. "Where We Came From." Gamasutra. 1 July2010.Web.27Feb.2012.

$<$ http://www.gamasutra.com/view/feature/5883/a_deeper_loo k_into_the_combat_php> 